\title{
Long-term effect of dietary fibre intake on glycosylated haemoglobin Alc level and glycaemic control status among Chinese patients with type 2 diabetes mellitus
}

\author{
Luxi Yang ${ }^{1}$, Le Shu ${ }^{1}$, Junyi Jiang ${ }^{2}$, Hua Qiu², Genming Zhao ${ }^{1}$, Yi Zhou ${ }^{2}$, \\ Qingwu Jiang ${ }^{1}$, Qiao Sun ${ }^{2}$, Guoyou Qin ${ }^{3}$, Hongyan $\mathrm{Wu}^{2}$, Liming Yang ${ }^{2}$, \\ Xiaonan Ruan ${ }^{2, *}$ and Wang Hong $X_{U}{ }^{1, *}$ \\ 'Department of Epidemiology, School of Public Health, Key Laboratory of Public Health Safety, Ministry of \\ Education, Fudan University, 138 Yi Xue Yuan Road, Shanghai 200032, People's Republic of China: \\ ${ }^{2}$ Department of Chronic Disease Prevention, Pudong New Area Centers for Disease Control and Prevention, \\ 3039 Zhang Yang Road, Shanghai 200136, People's Republic of China: ${ }^{3}$ Department of Biostatistics, \\ School of Public Health, Fudan University, Shanghai, People's Republic of China
}

Submitted 26 November 2012: Final revision received 5 June 2013: Accepted 12 June 2013: First published online 24 July 2013

\begin{abstract}
Objective: Dietary fibre has been linked to lower levels of glycosylated haemoglobin A1c (HbA1c) among diabetes patients. The present study aimed to evaluate the long-term effect of dietary fibre on HbA1c levels among Chinese patients with type 2 diabetes mellitus.

Design: Two cross-sectional surveys were conducted in 2006 and 2011, with the second one being a repeat survey on a sub-sample from the initial one. In both surveys, an in-person interview was conducted to collect information on demographic characteristics and lifestyles following a similar protocol. Dietary intake was assessed with a validated FFQ. Anthropometric measures and biochemical assays were performed at the interview.

Setting: Communities in Pudong New Area of Shanghai, China.

Subjects: Chinese patients ( $n$ 934) with type 2 diabetes mellitus.

Results: An inverse association was observed between dietary fibre and glycaemic status indicated by HbA1c level in both surveys, although it was significant only in the first survey. Among 497 patients participating in both surveys, dietary fibre intake at the first survey was inversely associated with uncontrolled glycaemic status at the second survey, with adjusted odds ratios across the tertiles of intake being $1 \cdot 00,0 \cdot 72(95 \%$ CI $0 \cdot 43,1 \cdot 21)$ and $0 \cdot 58\left(95 \%\right.$ CI 0.34, 0.99; $\left.P_{\text {trend }}=0 \cdot 048\right)$. The change in fibre intake was slightly associated with glycaemic status, with each increase in tertile scores of intake linked to a $0 \cdot 138 \%(\beta=-0 \cdot 138 ; 95 \%$ $\mathrm{CI}-0 \cdot 002,0 \cdot 278)$ decrease in HbA1c value and a $19 \%(\mathrm{OR}=0 \cdot 81 ; 95 \%$ CI $0 \cdot 65$, $1 \cdot 02)$ reduced risk of uncontrolled glycaemic status at the second survey. Conclusions: Dietary fibre may have a long-term beneficial effect on HbA1c level among Chinese diabetes patients.
\end{abstract}

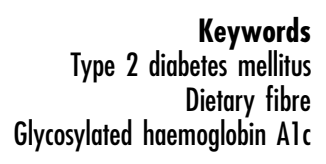

Type 2 diabetes mellitus (T2DM) is a chronic disease mainly characterized by the disorder of glucose metabolism, which may lead to a variety of specific severe complications such as CHD, stroke, diabetic retinopathy and kidney failure ${ }^{(1)}$. T2DM and its complications have been becoming a main burden of disease around the world, particularly in developing countries ${ }^{(2)}$. Aggressive control of hyperglycaemia, either by medicine or lifestyle intervention, is crucial to decrease the incidence of diabetic complications and the related premature death ${ }^{(3,4)}$. As a biomarker reflecting an individual's average level of blood glucose over past 2-3 months, glycosylated haemoglobin A1c (HbA1c) has been associated with the risk of long-term diabetes complications ${ }^{(5)}$ and used as the main indicator of glycaemic control status among diabetes patients ${ }^{(6)}$. The American Diabetes Association recommends an $\mathrm{HbA} 1 \mathrm{c}$ level below $7 \cdot 0 \%$ as the goal of glycaemic control for diabetes patients ${ }^{(6)}$.

There is sufficient evidence to support the role of dietary fibre in improving glycaemic control status in diabetes patients ${ }^{(7-9)}$. Evidence is also available for its beneficial effect on prevention of diabetes complications. By following up 7822 US women with T2DM in the Nurses' Health Study for 26 years, He et al. ${ }^{(10)}$ found that 
the highest $v$. the lowest quintile of intakes of whole grains, cereal fibre, bran and germ were associated with $16 \%$ to $31 \%$ lower all-cause mortality. Bran intake was inversely associated with CVD-specific mortality. The US Department of Agriculture has recommended a minimum daily fibre intake of $3.35 \mathrm{~g} / 1000 \mathrm{~kJ}(14 \mathrm{~g} / 1000 \mathrm{kcal})$ for individuals at high risk for $\mathrm{T}_{2} \mathrm{DM}^{(11)}$. The American Diabetes Association also suggests that patients with T2DM should increase their consumption of dietary fibre to adjust blood sugar and blood lipids, and thus reduce the risk of diabetic complications ${ }^{(6)}$.

Compared with Western populations, Chinese people usually have more dietary fibre intake ${ }^{(12)}$. The average level of total and soluble fibre intakes in Chinese diabetes patients were reported to reach 26.5 and $10.4 \mathrm{~g} / \mathrm{d}$, respectively, in a small-scale hospital-based survey ${ }^{(13)}$, more than the moderate amount recommended by the American Diabetes Association (total $24 \mathrm{~g} / \mathrm{d}$; soluble fibre $8 \mathrm{~g} / \mathrm{d}$ and insoluble fibre $16 \mathrm{~g} / \mathrm{d})^{(6)}$. However, both glycaemic control status and complications control were unsatisfactory for Chinese diabetes patients ${ }^{(14)}$.

We previously reported an inverse association between dietary fibre and HbA1c among Chinese patients with $\mathrm{T}_{2} \mathrm{DM}^{(15)}$. Based on the initial cross-sectional study, we further conducted a repeat dietary survey, aimed to evaluate the long-term effect of dietary fibre intake on glycaemic status among Chinese patients with T2DM.

\section{Materials and methods}

\section{Participants}

As described in our previous report ${ }^{(15)}$, 979 prevalent patients with T2DM were recruited in the communities of Shanggang, Zhoujiadu, Huamu, Puxin, Weifang, Jinyang, Meiyuan and Jichang in Pudong New Area of Shanghai, China, during the period October to December 2006. These patients were diagnosed with T2DM by physicians according to the 1999 WHO diagnostic criteria: (i) fasting plasma glucose $\geq 7 \cdot 0 \mathrm{mmol} / \mathrm{l}$; or (ii) 2 -h plasma glucose $\geq 11.1 \mathrm{mmol} / \mathrm{l}$ during an oral glucose tolerance test $(75-\mathrm{g}$ glucose load should be used); or (iii) a random plasma glucose concentration $\geq 11 \cdot 1 \mathrm{mmol} / 1$ in persons with symptoms of hyperglycaemia or hyperglycaemic crisis. After excluding those occurring with a cardiovascular event during the previous 6 months, having advanced congestive heart failure, unstable angina, major depression or dementia, 934 eligible patients with a mean age of 64.5 (SD $10 \cdot 1)$ years were interviewed; $41.7 \%$ of these patients were male.

During the period May to July 2011, a repeat survey was conducted for all participants of the first survey using a similar protocol. A total of 508 patients were successfully followed up, with a response rate of $54 \cdot 4 \%$. Of the 426 individuals lost, eighty-one were deceased or heavily sick, seventy-seven were out of town, eighty-one declined and 187 could not be contacted.

\section{Data collection}

In both surveys, a face-to-face interview was conducted by well-trained interviewers using a structured questionnaire after obtaining written consent from each participant. The collected information included demographic characteristics, duration of being diagnosed with T2DM, regular exercise (at least three times per week and at least half an hour per time), dietary habits, oral hypoglycaemia drug use and insulin use, and T2DM in first- and second-degree relatives.

At the interview, body measurement was conducted to collect body height, weight, waist circumference and hip circumference for each participant by following a standardized protocol. BMI was defined as weight divided by height squared $\left(\mathrm{kg} / \mathrm{m}^{2}\right)$.

\section{Dietary assessment}

Dietary intake was assessed using an interview-administered FFQ. The FFQ was modified based on an FFQ whose validity and reproducibility had been evaluated in the same population ${ }^{(16)}$. The FFQ specifies 103 food items, covering $90 \%$ of food items commonly consumed by Shanghai citizens. Participants were asked to report the frequency (daily, weekly, monthly, annually or never) and duration (months per year) of their consumption of each food item, as well as the estimated amount for each time they ate in the unit of liang ( 1 liang $=50 \mathrm{~g}$ ). The amount of intake was reported in millilitres for liquid foods like milk, juice and beverages, and was further transformed to grams in data analysis. The daily intakes of oil, salt and sugar were calculated as the average level consumed by each member of the participant's family.

The Chinese Food Composition Tables were utilized to estimate the amount of nutrients from each food item and to obtain glycaemic index (GI) values for most food items $^{(17)}$. For the remaining GI values we referred to Foster-Powell et al.'s report ${ }^{(18)}$. Glycaemic load (GL) was calculated by multiplying a food's GI (as a percentage) by the net carbohydrates in a given serving ${ }^{(19)}$. The daily GL intake was calculated by summing the GL from each food item consumed, and the average GI intake was further obtained by dividing the daily GL intake by the total net carbohydrate intake. For dietary fibre intake, only insoluble fibre was included. We excluded from the study participants who had extreme values for total energy intake $(<3347$ or $>16736 \mathrm{~kJ} / \mathrm{d}(<800$ or $>4000 \mathrm{kcal} / \mathrm{d})$ for men, $n 5$; $<2092$ or $>14644 \mathrm{~kJ} / \mathrm{d}(<500$ or $>3500 \mathrm{kcal} / \mathrm{d})$ for women, $n 3$ ) to minimize the potential recall bias, as did previous studies ${ }^{(20)}$.

\section{Biochemical assay}

In both surveys, a blood sample for biochemical assay was collected from each participant after an overnight fast of at least $10 \mathrm{~h}$. As described in our previous report ${ }^{(15)}$, HbA1c was measured using ion-exchange chromatography on a DS5 Glycated Hemoglobin Analyzer (DREW DS5; 
Drew Scientific Co. Ltd, Cumbria, UK). Quality assessment of assays was performed internally and externally ${ }^{(15)}$.

\section{Statistical analysis}

Statistical analyses were conducted utilizing the SAS statistical software package version 9.3. Differences in characteristics and dietary factors between the two surveys were compared using $\chi^{2}$ tests for categorical variables and non-parametric signed-rank tests for continuous variables. Pearson correlation tests were used to evaluate the consistency of the two measures of dietary fibre intake per $1000 \mathrm{~kJ} / \mathrm{d}$. An unconditional logistic regression model was applied to estimate the adjusted odds ratios and $95 \%$ confidence intervals of dietary fibre intake per $1000 \mathrm{~kJ} / \mathrm{d}$ with glycaemic control status, which was classified as 'controlled' and 'uncontrolled' by HbA1c level of $7 \cdot 0 \%$. Dietary fibre intake per $1000 \mathrm{~kJ} / \mathrm{d}$ was classified into tertile groups and scored as 1,2 and 3 by sex-specific cut-off points for the first and second surveys, respectively. The change in dietary fibre intake was specifically defined as the difference in tertile scores between the two surveys, and thus yielded five groups ranked as $-2,-1,0,1$ and 2. A generalized linear model was used to estimate the effect of changes in dietary fibre intake on the difference of HbA1c level between the two surveys. All statistical tests were based on two-sided probability and the significance level was $0 \cdot 05$.

\section{Results}

Of a total of 934 participants, 508 patients were successfully followed up and 497 donated a blood sample. Compared with the non-participants of the second survey, the participants were significantly younger and less likely to use an oral hypoglycaemia drug and insulin at the first survey. However, no significant difference was observed between the two groups with regard to sex, BMI, family history of diabetes, duration of diabetes and presence of other chronic diseases (data not shown).

Among the 508 patients who participated in both surveys, more family history of diabetes, higher prevalence of hypertension, dyslipidaemia and CHD, more use of an oral hypoglycaemia drug and/or insulin, a higher level of dietary fat but lower levels of energy, carbohydrate, dietary GI and dietary fibre intakes were observed at the second survey than at the first survey, as shown in Table 1 . These patients, however, were more likely to exercise and had lower average BMI at the second survey. No significant difference was observed for intakes of energy-adjusted

Table 1 Characteristics and dietary intake in the two surveys: Chinese patients with T2DM, Pudong New Area of Shanghai (two crosssectional surveys were conducted in 2006 and 2011)

\begin{tabular}{|c|c|c|c|c|c|c|c|}
\hline & & & \multicolumn{4}{|c|}{ Participants of both surveys ( $n 508)$} & \multirow[b]{3}{*}{$P$ value } \\
\hline & \multicolumn{2}{|c|}{$\begin{array}{l}\text { Participants of first survey } \\
\text { (n 934) }\end{array}$} & \multicolumn{2}{|c|}{ At the first survey } & \multicolumn{2}{|c|}{ At the second survey } & \\
\hline & Mean or $n$ & SD or $\%$ & Mean or $n$ & SD or $\%$ & Mean or $n$ & SD or $\%$ & \\
\hline \multicolumn{8}{|l|}{ Characteristic } \\
\hline Age (years)t & $64 \cdot 5$ & $10 \cdot 1$ & $63 \cdot 8$ & $9 \cdot 2$ & $68 \cdot 4$ & $9 \cdot 2$ & - \\
\hline Male gender, $n(\%)$ & 389 & $41 \cdot 7$ & 201 & $39 \cdot \overline{6}$ & 201 & $39 \cdot \overline{6}$ & - \\
\hline Family history of T2DM, $n(\%)$ & 298 & 31.9 & 174 & $34 \cdot 3$ & 209 & $42 \cdot 2$ & $<0.001$ \\
\hline Age of T2DM diagnosis (years) $\dagger$ & $55 \cdot 2$ & $10 \cdot 4$ & $54 \cdot 8$ & $9 \cdot 7$ & $54 \cdot 8$ & $9 \cdot \overline{7}$ & - \\
\hline Duration of T2DM (years)t & $9 \cdot 2$ & $6 \cdot 4$ & $9 \cdot 0$ & $5 \cdot 9$ & 13.5 & $5 \cdot 9$ & - \\
\hline BMI $\left(\mathrm{kg} / \mathrm{m}^{2}\right) \dagger$ & $25 \cdot 8$ & $3 \cdot 6$ & $25 \cdot 8$ & $3 \cdot 6$ & $25 \cdot 5$ & $3 \cdot 6$ & 0.001 \\
\hline Hypertension, $n(\%)$ & 518 & $55 \cdot 5$ & 281 & $55 \cdot 3$ & 304 & $59 \cdot 8$ & 0.034 \\
\hline Dyslipidaemia, $n(\%)$ & 97 & $10 \cdot 4$ & 56 & $11 \cdot 0$ & 83 & $16 \cdot 3$ & 0.006 \\
\hline CVD, $n(\%)$ & 132 & $14 \cdot 1$ & 70 & $13 \cdot 8$ & 200 & $19 \cdot 7$ & 0.002 \\
\hline Hypoglycaemia drug use, yes, $n(\%)$ & 762 & $81 \cdot 9$ & 433 & $85 \cdot 2$ & 386 & $78 \cdot 5$ & $<0.001$ \\
\hline Insulin use, yes, $n(\%)$ & 88 & $9 \cdot 5$ & 35 & $6 \cdot 9$ & 123 & $25 \cdot 0$ & $<0.001$ \\
\hline \multirow[t]{2}{*}{ Exercise, yes, $n(\%)$} & 434 & $46 \cdot 5$ & 247 & $48 \cdot 6$ & 280 & $56 \cdot 7$ & 0.007 \\
\hline & Median & $\begin{array}{l}\text { 25th, } 75 \text { th } \\
\text { percentile }\end{array}$ & Median & $\begin{array}{l}\text { 25th, } 75 \text { th } \\
\text { percentile }\end{array}$ & Median & $\begin{array}{l}\text { 25th, } 75 \text { th } \\
\text { percentile }\end{array}$ & \\
\hline \multicolumn{8}{|l|}{ Dietary intake } \\
\hline Energy $(\mathrm{kJ} / \mathrm{d})$ & 6372 & 5268,7594 & 6322 & 5188,7665 & 4954 & 3987,6113 & $<0.001$ \\
\hline Dietary fibre $(\mathrm{g} / \mathrm{d})$ & $8 \cdot 7$ & $6 \cdot 2,12 \cdot 6$ & $8 \cdot 7$ & $6 \cdot 0,13 \cdot 0$ & 6.9 & $4 \cdot 8,10 \cdot 1$ & $<0.001$ \\
\hline Dietary fibre $(\mathrm{g} / 1000 \mathrm{~kJ}$ per $\mathrm{d})$ & $1 \cdot 39$ & $1 \cdot 00,1 \cdot 82$ & $1 \cdot 39$ & $1 \cdot 00,1 \cdot 84$ & $1 \cdot 31$ & $0.98,1.82$ & 0.252 \\
\hline Fat $(\%$ of energy) & $21 \cdot 1$ & $16 \cdot 8,25 \cdot 7$ & $22 \cdot 1$ & $16 \cdot 9,26 \cdot 2$ & $40 \cdot 8$ & $34 \cdot 1,49 \cdot 6$ & $<0.001$ \\
\hline Protein ( $\%$ of energy) & $14 \cdot 7$ & $12 \cdot 8,16 \cdot 8$ & $14 \cdot 5$ & $13 \cdot 0,16 \cdot 6$ & $15 \cdot 1$ & $12 \cdot 0,17 \cdot 5$ & 0.852 \\
\hline Carbohydrate (\% of energy) & $63 \cdot 4$ & $58 \cdot 1,69 \cdot 6$ & $62 \cdot 8$ & $57 \cdot 1,68 \cdot 6$ & 43.0 & $35 \cdot 8,49 \cdot 6$ & $<0.001$ \\
\hline Dietary Gl & $60 \cdot 1$ & 54,66 & $60 \cdot 2$ & 54,66 & $35 \cdot 5$ & 30,41 & $<0.001$ \\
\hline Dietary GL & $81 \cdot 6$ & 65,100 & $81 \cdot 1$ & 64,100 & $79 \cdot 4$ & 62,101 & 0.888 \\
\hline
\end{tabular}

T2DM, type 2 diabetes mellitus; GI, glycaemic index; GL, glycaemic load.

${ }^{*}$ Paired $\chi^{2}$ test or signed-rank test.

+Values are presented as mean and standard deviation. 
Table 2 Association of dietary fibre intake with glycaemic control status in Chinese patients with T2DM, Pudong New Area of Shanghai (two cross-sectional surveys were conducted in 2006 and 2011)

\begin{tabular}{|c|c|c|c|c|c|c|c|c|c|}
\hline \multirow{3}{*}{$\begin{array}{l}\text { Dietary fibre intake } \\
(\mathrm{g} / 1000 \mathrm{~kJ} \text { per } \mathrm{d})^{\star}\end{array}$} & & & \multicolumn{4}{|c|}{ Glycaemic control status } & \multirow[b]{3}{*}{$\mathrm{ORt}, \ddagger$} & \multirow[b]{3}{*}{$95 \% \mathrm{Cl}$} & \multirow[b]{3}{*}{$P$ for trenc } \\
\hline & \multicolumn{2}{|r|}{$\mathrm{HbA1c}(\%)$} & \multicolumn{2}{|c|}{$\begin{array}{c}\text { Controlled } \\
(\mathrm{HbA} 1 \mathrm{c} \leq 7 \%)\end{array}$} & \multicolumn{2}{|c|}{$\begin{array}{l}\text { Uncontrolled } \\
(\mathrm{HbA} 1 \mathrm{c}>7 \%)\end{array}$} & & & \\
\hline & Median & 25th, 75th percentile & $n$ & $\%$ & $n$ & $\%$ & & & \\
\hline \multicolumn{10}{|c|}{ At first survey } \\
\hline \multicolumn{10}{|c|}{ At first survey ( $n$ 934) } \\
\hline Low & $7 \cdot 4$ & $6 \cdot 6,8 \cdot 7$ & 126 & $27 \cdot 9$ & 187 & $38 \cdot 7$ & $1 \cdot 00$ & - & \\
\hline Medium & $6 \cdot 9$ & $6 \cdot 4,8 \cdot 2$ & 160 & $35 \cdot 5$ & 150 & $31 \cdot 1$ & 0.62 & $0.44,0.87$ & \\
\hline High & $7 \cdot 0$ & $6 \cdot 2,8 \cdot 1$ & 165 & $36 \cdot 6$ & 146 & $30 \cdot 2$ & 0.54 & $0.37,0.77$ & $<0.001$ \\
\hline \multicolumn{10}{|c|}{ At second survey } \\
\hline \multicolumn{10}{|c|}{ At first survey ( $n$ 497) } \\
\hline Low & $7 \cdot 4$ & $6 \cdot 3,8 \cdot 6$ & 76 & $26 \cdot 8$ & 90 & $42 \cdot 3$ & $1 \cdot 00$ & - & \\
\hline Medium & $6 \cdot 7$ & $5 \cdot 9,7 \cdot 7$ & 100 & $35 \cdot 2$ & 65 & $30 \cdot 5$ & 0.72 & $0 \cdot 43,1 \cdot 21$ & \\
\hline High & $6 \cdot 5$ & $5 \cdot 5,7 \cdot 6$ & 108 & $38 \cdot 0$ & 58 & $27 \cdot 2$ & 0.58 & $0.34,0.99$ & 0.048 \\
\hline \multicolumn{10}{|c|}{ At second survey } \\
\hline At second survey ( & & & & & & & & & \\
\hline Low & $7 \cdot 1$ & $5 \cdot 9,8 \cdot 2$ & 81 & $28 \cdot 9$ & 86 & $39 \cdot 8$ & $1 \cdot 00$ & - & \\
\hline Medium & $6 \cdot 6$ & $5 \cdot 8,7 \cdot 8$ & 104 & $37 \cdot 1$ & 60 & $27 \cdot 8$ & 0.58 & $0.36,0.93$ & \\
\hline High & $6 \cdot 8$ & $5 \cdot 9,8 \cdot 0$ & 95 & 33.9 & 70 & $32 \cdot 4$ & 0.75 & $0 \cdot 46,1 \cdot 21$ & $0 \cdot 261$ \\
\hline
\end{tabular}

T2DM, type 2 diabetes mellitus; HbA1c, glycosylated haemoglobin A1c.

*The tertile cut-off points of dietary fibre intake at the first survey for all 934 participants were respectively 1.04 and $1.58 \mathrm{~g} / 1000 \mathrm{~kJ}$ per $\mathrm{d}(4.35$ and $6.64 \mathrm{~g} /$ $1000 \mathrm{kcal}$ per d) in men and 1.19 and $1.65 \mathrm{~g} / 1000 \mathrm{~kJ}$ per $\mathrm{d}(4.97$ and $6.92 \mathrm{~g} / 1000 \mathrm{kcal}$ per d) in women; and for the 497 participants at the first survey were respectively 1.02 and $1.61 \mathrm{~g} / 1000 \mathrm{~kJ}$ per d $(4.26$ and $6.75 \mathrm{~g} / 1000 \mathrm{kcal}$ per d) in men and 1.18 and $1.66 \mathrm{~g} / 1000 \mathrm{~kJ}$ per d $(4.96 \mathrm{and} 6.94 \mathrm{~g} / 1000 \mathrm{kcal}$ per d) in women; the tertile cut-off points of dietary fibre intake at the second survey for the 497 participants were 1.04 and $1.50 \mathrm{~g} / 1000 \mathrm{~kJ}$ per $\mathrm{d}(4.37$ and $6.30 \mathrm{~g} /$ $1000 \mathrm{kcal}$ per d) in men and 1.12 and $1.72 \mathrm{~g} / 1000 \mathrm{~kJ}$ per $\mathrm{d}(4.67$ and $7 \cdot 19 \mathrm{~g} / 1000 \mathrm{kcal}$ per d) in women.

†Adjusted for age (continuous variable), gender (male/female), BMI (continuous variable), duration of T2DM (continuous variable), physical activity (active/ inactive), drug use (no drug/hypoglycaemia drug only/insulin use, dummy variables), family history of diabetes (yes/no), carbohydrate intake per $1000 \mathrm{~kJ} / \mathrm{d}$ (continuous variable) and energy intake (continuous variable).

$\ddagger$ The $\mathrm{HbA1c}$ level in the first survey (continuous variable) is adjusted for the association of dietary fibre intake per $1000 \mathrm{~kJ} / \mathrm{d}$ at the first survey and glycaemic control status at the second survey.

dietary fibre $(P=0 \cdot 252)$, protein $(P=0 \cdot 852)$ and dietary GL $(P=0 \cdot 888)$ between the two surveys.

Although dietary fibre intake at the second survey was significantly lower than that at the first one, the two measures of daily intake per $1000 \mathrm{~kJ}$ were significantly correlated with each other, with a Pearson correlation coefficient of $0 \cdot 100(P=0 \cdot 028)$.

As shown in Table 2, an inverse association of dietary fibre with glycaemic control status was observed in both cross-sectional surveys, with adjusted OR of uncontrolled glycaemic status across the tertiles of fibre intake being $1 \cdot 00,0.62(95 \%$ CI $0.44,0.87)$ and $0.54(95 \%$ CI 0.37 , $0.77)$ at the first survey $\left(P_{\text {trend }}<0.001\right)$ and $1.00,0.58$ (95\% CI $0 \cdot 36,0 \cdot 93)$ and $0 \cdot 75$ (95\% CI $0 \cdot 46,1 \cdot 21)$ at the second survey $\left(P_{\text {trend }}=0 \cdot 261\right)$. Among 497 patients donating bio-specimens in both surveys, dietary fibre intake at the first survey was inversely associated with uncontrolled glycaemic status at the second survey. Compared with patients in the lowest tertile of intake, the adjusted $\mathrm{OR}$ of uncontrolled glycaemic status was reduced from $0.72(95 \% \mathrm{CI} 0 \cdot 43,1 \cdot 21)$ to $0.58(95 \% \mathrm{CI}$ $0.34,0.99)$ with increasing tertile of dietary fibre intake $\left(P_{\text {trend }}=0 \cdot 048\right)$. No similar association pattern was observed for GI or GL intake (data not shown in the table).

Figure 1 presents the association of dietary fibre and HbA1c level by duration of T2DM. Generally, regardless of the duration of T2DM, patients with higher fibre intake had a lower level of HbA1c. The long-term effect of dietary fibre intake appeared more pronounced than the recent effect (Fig. 1(c) v. Fig. 1(a) and Fig. 1(b)) and the effect was more evident among patients with longer T2DM duration (Fig. 1(c)).

We further evaluated the association of the changes in dietary fibre intake with the changes in HbA1c level. As shown in Table 3, a borderline association was observed between the change in dietary fibre intake and HbA1c change. Specifically, each increase in tertile scores of dietary fibre intake was associated with a $0 \cdot 138 \%$ decrease in HbA1c level $(\beta=-0 \cdot 138 ; 95 \%$ CI $-0 \cdot 002,0 \cdot 278$; $P=0.054)$ and a $19 \%(\mathrm{OR}=0.81 ; 95 \%$ CI $0 \cdot 65,1 \cdot 02)$ reduced risk of uncontrolled glycaemic status at the second survey $\left(P_{\text {trend }}=0 \cdot 070\right)$. The OR of uncontrolled glycaemic status were $1 \cdot 16(95 \% \mathrm{CI} 0 \cdot 63,2 \cdot 13)$ and $1 \cdot 71$ (95\% CI 0.82, 3.60) for those with -1 and -2 decrease in tertile scores of fibre intake, and $0.58(95 \% \mathrm{CI} 0 \cdot 33,1 \cdot 00)$ and $1 \cdot 15$ (95\% CI $0 \cdot 52,2 \cdot 54)$ for those with +1 and +2 increase in tertile scores, compared with those without change.

\section{Discussion}

In the present study with two repeat surveys among Chinese patients with T2DM, higher dietary fibre intake was consistently associated with better glycaemic control 
(a)

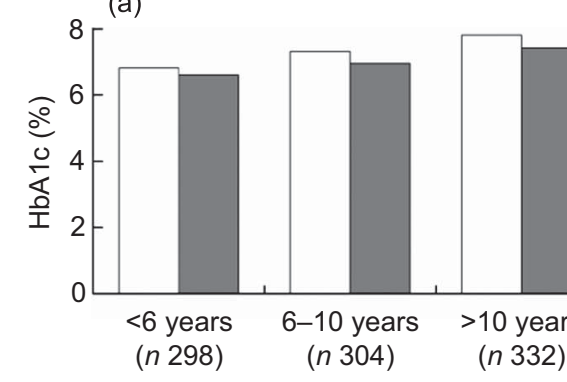

(b)

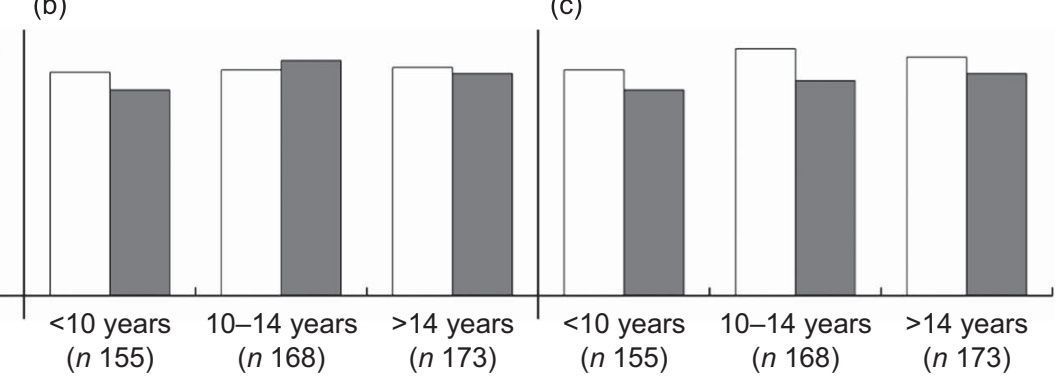

Fig. 1 Average level of glycosylated haemoglobin A1c (HbA1c) by dietary fibre intake ( $\square$, low fibre intake; $\square$, high fibre intake) among Chinese patients with different duration of type 2 diabetes mellitus (T2DM), Pudong New Area of Shanghai (two crosssectional surveys were conducted in 2006 and 2011). All $P$ values from logistic regression models were adjusted for age (continuous variable), gender (male/female), BMI (continuous variable), regular exercise (ever/ never), drug use (no drug/ hypoglycaemia drug only/insulin use, dummy variables), family history of diabetes (yes/no), carbohydrate intake per $1000 \mathrm{~kJ} / \mathrm{d}$ (continuous variable) and energy intake (continuous variable). $P$ values were: $0.054,0.054$ and $0 \cdot 115$, respectively, for patients diagnosed with T2DM for $<6$ years, $6-10$ years and $>10$ years in (a); 0.045, 0.385 and 0.069 , respectively, for patients diagnosed with T2DM for $<10$ years, 10-14 years and $>14$ years in (b); and $0.332,0.032$ and 0.088 , respectively, for patients diagnosed with T2DM for $<10$ years, 10-14 years and $>14$ years in (c). In (a), both dietary fibre and HbA1c level were measured at the first survey; the median cut-off point for dietary fibre was $1.28 \mathrm{~g} / 1000 \mathrm{~kJ}$ per $\mathrm{d}(5.37 \mathrm{~g} / 1000 \mathrm{kcal}$ per d) for men and $1.42 \mathrm{~g} / 1000 \mathrm{~kJ}$ per $\mathrm{d}$ $(5.94 \mathrm{~g} / 1000 \mathrm{kcal}$ per d) for women. In (b), both dietary fibre and HbA1c level were measured at the second survey; the median cutoff point for dietary fibre was $1.24 \mathrm{~g} / 1000 \mathrm{~kJ}$ per d $(5 \cdot 20 \mathrm{~g} / 1000 \mathrm{kcal}$ per d) for men and $1.36 \mathrm{~g} / 1000 \mathrm{~kJ}$ per d $(5 \cdot 70 \mathrm{~g} / 1000 \mathrm{kcal}$ per d) for women. In (c), dietary fibre was measured at the first survey and $\mathrm{HbA1c}$ level was assayed at the second survey; the median cut-off point for dietary fibre was $1.25 \mathrm{~g} / 1000 \mathrm{~kJ}$ per d $(5 \cdot 24 \mathrm{~g} / 1000 \mathrm{kcal}$ per d) for men and $1.44 \mathrm{~g} / 1000 \mathrm{~kJ}$ per d $(6.03 \mathrm{~g} / 1000 \mathrm{kcal}$ per d) for women

status and showed a long-term protective effect on HbA1c level. To the best of our knowledge, the present study is the first that evaluates the effect of changes in dietary fibre intake on glycaemic status among Chinese diabetes patients. Our results provide preliminary evidence on the beneficial effect of fibre intake on glycaemic control and implicate the potential role of dietary fibre intake in prevention of diabetic complications in this population.

Dietary fibre has been shown to benefit human health in several ways ${ }^{(11)}$. The mechanism by which dietary fibre reduces the risk of T2DM and diabetes complications is not clear, however. It is suggested that a fibre-rich diet provides limited energy ${ }^{(21)}$, has longer processing time in stomach $^{(22)}$ and a low GI, which are considered the primary way for dietary fibre to affect glycaemic control ${ }^{(23,24)}$. Numerous experimental studies have indicated that viscous dietary fibres benefit immediate postprandial glucose metabolism and long-term glycaemic control ${ }^{(7,25)}$.

Chinese adults usually consume more dietary fibre compared with their counterparts in other countries and regions ${ }^{(12)}$. It is reported that dietary fibre intake among Chinese T2DM patients reaches the recommended level for Americans ${ }^{(13)}$. In the present study focusing on insoluble fibre intake only, we found that the median intake of dietary fibre was $8.7 \mathrm{~g} / \mathrm{d}(1.39 \mathrm{~g} / 1000 \mathrm{~kJ}$ per $\mathrm{d}$, i.e. $5 \cdot 8 \mathrm{~g} / 1000 \mathrm{kcal}$ per d) at the first survey and $6 \cdot 9 \mathrm{~g} / \mathrm{d}$ $(1.31 \mathrm{~g} / 1000 \mathrm{~kJ}$ per d, i.e. $5.5 \mathrm{~g} / 1000 \mathrm{kcal}$ per d) at the second survey, which is less than the reported average intake in healthy Chinese adults ${ }^{(12,26)}$ and below the recommended level of $16 \mathrm{~g} / \mathrm{d}(1.91 \mathrm{~g} / 1000 \mathrm{~kJ}$ per d, i.e. $8 \mathrm{~g} / 1000 \mathrm{kcal}$ per d) for adults at high risk of T2 $\mathrm{DM}^{(6)}$.
Our study suggests that dietary fibre intake among Chinese diabetes patients may have been overestimated, although the data derived from that single study were not representative $^{(13)}$. Due to the lower average level of energy intake, however, it is also possible that the absolute intake of fibre was underestimated in the current study.

In the present study, the two measurements of dietary fibre intake were significantly correlated, although with a small correlation coefficient. Moreover, most participants had no or a \pm 1 score change in tertile scores between two surveys. These results indicate relatively stable dietary habits along the duration of the disease in patients. In both cross-sectional surveys and the small-scale prospective investigation, we consistently observed an inverse association between dietary fibre and glucose control status. We also observed an insignificant association of changes in dietary fibre intake with changes in HbA1c level. Interestingly, we found that the beneficial effect of dietary fibre in this patient population was slightly more pronounced among those with longer T2DM duration. These findings implicate a potential longterm protective effect of dietary fibre in Chinese diabetes patients and indicate a possible clinical significance of increasing dietary fibre intake in these patients.

In this population of patients, we did not observe a significant association of dietary GI and GL intake with glycaemic status, which was not consistent with previous studies $^{(8,27,28)}$. Due to the generally high correlation between dietary fibre and GI/GL, the null association of GI/GL with glycaemic status in this population is somewhat difficult to explain. Our results appeared not to 
support the notion that low GI or GL value is the primary way for dietary fibre to affect glycaemic control, and suggest mechanisms other than GI value. The inaccurate GI value for some Chinese foods may also partly explain the results.

There are several strengths in the present study. First of all, two repeat surveys following a similar protocol make it possible to evaluate the long-term effect of dietary fibre intake on glycaemic control status. The validated FFQ, a standardized protocol for body measurements and stringent quality control in laboratory assays on HbA1c level minimized the potential information bias.

However, the high dropout rate (45.6\%) in the second survey may have introduced selection bias and decreased the validity of our results. The participants of the second survey were older and more likely to use an oral hypoglycaemia drug and insulin, indicating that they had more severe disease or uncontrolled HbA1c level. Whether dietary fibre intake can elicit a long-term protective effect on these severe patients remains unknown. Moreover, the amount of dietary intake in both surveys seemed much lower than those reported previously ${ }^{(26)}$, raising our concern on the possible recall bias. However, the potential underestimation of dietary intake, if any, would be more likely to result in a non-differential misclassification bias, which may have biased our results towards the null. Furthermore, most nutrients were significantly lower in the second survey than in the first one, which may be due to ageing of the patients and the progression of T2DM. However, recall bias cannot be excluded. In addition, the two surveys were conducted in different seasons, which may affect the results and partly explain the lower dietary fibre intake but higher fat intake in the second survey. It is also possible that generally the Chinese middle-aged and elderly residents took less plant foods and more animal foods, which led to a higher energy intake from fat ${ }^{(29)}$. To minimize the recall bias, we used energy-adjusted dietary fibre intake in the analysis and defined the changes in dietary fibre intake between the two surveys specifically as the difference in tertile scores instead of the difference in amount of intake.

In summary, our study provides suggestive evidence on the beneficial effect of dietary fibre on glycaemic control among Chinese diabetes patients. A well-designed cohort study or a randomized controlled trial is warranted to confirm our findings.

\section{Acknowledgements}

Sources of funding: This study was funded by the Shanghai Municipal Health Bureau (grant numbers 12GWZX1010 and GWDTR201204), the Academic Leaders Training Program of Pudong Health Bureau of Shanghai (grant number PWRd2010-03), and FDUROP project of Fudan University. The funders had no role in the design, 
analysis or writing of this article. Conflicts of interest: All co-authors declare that they have no competing interests. Ethics: The study was approved by Fudan University Institutional Review Board (IRB00002408, FWA00002399). Authors' contributions: L.X.Y. and L.S. contributed to data collection, data analysis and drafting of the paper. J.Y.J., H.Q., Y.Z., H.Y.W. and L.M.Y. contributed to data collection and quality control. G.Y.Q. contributed to data analysis. G.M.Z., Q.W.J. and Q.S. contributed to revision of the paper. X.N.R. and W.H.X. contributed to study design, statistical analysis and revision of the paper. Acknowledgements: The authors are grateful to the study participants and research staff from Community Health Centers in Pudong New Area of Shanghai, China.

\section{References}

1. van Dieren S, Beulens JW, van der Schouw YT et al. (2010) The global burden of diabetes and its complications: an emerging pandemic. Eur J Cardiovasc Prev Rehabil 17, Suppl. 1, S3-S8.

2. Herman WH \& Zimmet P (2012) Type 2 diabetes: an epidemic requiring global attention and urgent action. Diabetes Care 35, 943-944.

3. Ismail-Beigi F, Craven T, Banerji MA et al. (2010) Effect of intensive treatment of hyperglycaemia on microvascular outcomes in type 2 diabetes: an analysis of the ACCORD randomised trial. Lancet 376, 419-430.

4. Ray KK, Seshasai SR, Wijesuriya S et al. (2009) Effect of intensive control of glucose on cardiovascular outcomes and death in patients with diabetes mellitus: a meta-analysis of randomised controlled trials. Lancet 373, 1765-1772.

5. Stratton IM, Adler AI, Neil HA et al. (2000) Association of glycaemia with macrovascular and microvascular complications of type 2 diabetes (UKPDS 35): prospective observational study. BMJ 321, 405-412.

6. American Diabetes Association (2012) Standards of medical care in diabetes - 2012. Diabetes Care 35, Suppl. 1, S11-S63.

7. Chandalia M, Garg A, Lutjohann D et al. (2000) Beneficial effects of high dietary fiber intake in patients with type 2 diabetes mellitus. N Engl J Med 342, 1392-1398.

8. Jenkins DJ, Kendall CW, McKeown-Eyssen G et al. (2008) Effect of a low-glycemic index or a high-cereal fiber diet on type 2 diabetes: a randomized trial. JAMA 300, 2742-2753.

9. Post RE, Mainous AG 3rd, King DE et al. (2012) Dietary fiber for the treatment of type 2 diabetes mellitus: a metaanalysis. J Am Board Fam Med 25, 16-23.

10. He M, van Dam RM, Rimm E et al. (2010) Whole-grain, cereal fiber, bran, and germ intake and the risks of all-cause and cardiovascular disease-specific mortality among women with type 2 diabetes mellitus. Circulation 121, 2162-2168.

11. Institute of Medicine, Food and Nutrition Board (2005) Dietary, functional, and total fiber. In Dietary Reference Intakes for Energy, Carbohydrate, Fiber, Fat, Fatty Acids, Cholesterol, Protein, and Amino Acids (Macronutrients), pp. 339-421. Washington, DC: The National Academies Press.
12. Zhou BF, Stamler J, Dennis B et al. (2003) Nutrient intakes of middle-aged men and women in China, Japan, United Kingdom, and United States in the late 1990s: the INTERMAP study. J Hum Hypertens 17, 623-630.

13. Yin WY, Zheng WD, Huang CY et al. (2005) Investigation of dietary fiber intakes and varies in 53 patients with diabetes. Zhonghua Yu Fang Yi Xue Za Zhi 39, 342-344.

14. Liu Z, Fu C, Wang W et al. (2010) Prevalence of chronic complications of type 2 diabetes mellitus in outpatients - a cross-sectional hospital based survey in urban China. Health Qual Life Outcomes 8, 62.

15. Jiang JY, Qiu H, Zhao GM et al. (2012) Dietary fibre intake is associated with HbA1c level among prevalent patients with type 2 diabetes in Pudong New Area of Shanghai, China. PLoS One 7, e 46552.

16. Shu XO, Yang G, Jin F et al. (2004) Validity and reproducibility of the food frequency questionnaire used in the Shanghai Women's Health Study. Eur J Clin Nutr 58, $17-23$.

17. Yang YX, Wang GY \& Pan XC (2002) China Food Composition Tables 2002. Beijing: Beijing University Medical Press.

18. Foster-Powell K, Holt SH \& Brand-Miller JC (2002) International table of glycemic index and glycemic load values: 2002. Am J Clin Nutr 76, 5-56.

19. Pi-Sunyer FX (2002) Glycemic index and disease. Am J Clin Nutr 76, issue 1, 290S-298S.

20. Fung TT, Schulze M, Manson JE et al. (2004) Dietary patterns, meat intake, and the risk of type 2 diabetes in women. Arch Intern Med 164, 2235-2240.

21. Rolls BJ, Bell EA, Castellanos VH et al. (1999) Energy density but not fat content of foods affected energy intake in lean and obese women. Am J Clin Nutr 69, 863-871.

22. Jenkins DJ, Kendall CW, Augustin LS et al. (2002) Highcomplex carbohydrate or lente carbohydrate foods? Am J Med 113, Suppl. 9B, 30S-37S.

23. Jenkins AL, Jenkins DJ, Zdravkovic U et al. (2002) Depression of the glycemic index by high levels of $\beta$-glucan fiber in two functional foods tested in type 2 diabetes. Eur J Clin Nutr 56, 622-628.

24. Marangoni F \& Poli A (2008) The glycemic index of bread and biscuits is markedly reduced by the addition of a proprietary fiber mixture to the ingredients. Nutr Metab Cardiovasc Dis 18, 602-605.

25. Anderson JW, Allgood LD, Turner J et al. (1999) Effects of psyllium on glucose and serum lipid responses in men with type 2 diabetes and hypercholesterolemia. Am J Clin Nutr 70, 466-473.

26. Villegas R, Yang G, Gao YT et al. (2010) Dietary patterns are associated with lower incidence of type 2 diabetes in middle-aged women: the Shanghai Women's Health Study. Int J Epidemiol 39, 889-899.

27. Thomas DE \& Elliott EJ (2010) The use of low-glycaemic index diets in diabetes control. Br J Nutr 104, 797-802.

28. Esposito K, Maiorino MI, Di Palo C et al. (2010) Dietary glycemic index and glycemic load are associated with metabolic control in type 2 diabetes: the CAPRI experience. Metab Syndr Relat Disord 8, 255-261.

29. Su C, Wang H, Wang $Z$ et al. (2013) Status and trend of fat and cholesterol intake among Chinese middle and old aged residents in 9 provinces from 1991 to 2009. Wei Sheng Yan Jiu 42, 72-77. 